\title{
Determination of the Stabilization Time of the Solution-Air Interface for Aggregates Formed by NaC in Mixtures with SDS and PE0, Investigated by Dynamic Surface Tension Measurements
}

\author{
Grasiele Rauppㅁ, Arlindo C. Felippe ${ }^{2}$, Tiago Elias Allievi Frizon'1, Luciano da Silva1, \\ Marcos Marques da Silva Paula ${ }^{1}$, Alexandre G. Dal-Bó ${ }^{1}$ \\ ${ }^{1}$ Universidade do Extremo Sul Catarinense (UNESC), Criciúma, Brazil \\ ${ }^{2}$ Universidade Federal da Fronteira Sul (UFFS), Chapecó, Brazil \\ Email: alexandredalbo@hotmail.com
}

Received 17 December 2013; revised 5 January 2014; accepted 13 February 2014

Copyright $@ 2014$ by authors and Scientific Research Publishing Inc.

This work is licensed under the Creative Commons Attribution International License (CC BY).

http://creativecommons.org/licenses/by/4.0/

\section{(c) (i) Open Access}

\begin{abstract}
The properties of mixtures of poly (ethylene oxide) (PEO) and mixed micelles formed from sodium cholate ( $\mathrm{NaC}$ ) and sodium dodecyl sulfate (SDS) in tris/HCl buffered solutions at pH 9.00 were investigated by measuring the mean surface tension. The variation in the superficial tension as a function of the time after formation of solutions containing PEO and NaC was characterized by monitoring the time required for the system to reach equilibrium between the micellar and aqueous phases. These results could serve as a reference for the minimum aging time required for solutions before any surface tension measurements can be performed.
\end{abstract}

Keywords

Poly (Ethylene Oxide); Micelles; Sodium Cholate; Sodium Dodecyl Sulfate

\section{Introduction}

The interactions between surfactants and neutral water-soluble polymers have been the focus of intense investigation over the last decade [1]-[8]. The motivation for this research was identifying the physical-chemical properties of the solutions and any possible mechanisms that describe the bonding of the surfactant to the polymer.

How to cite this paper: Raupp, G. (2014) Determination of the Stabilization Time of the Solution-Air Interface for Aggregates Formed by NaC in Mixtures with SDS and PEO, Investigated by Dynamic Surface Tension Measurements. Soft, 3, 1-10. http://dx.doi.org/10.4236/soft.2014.31001 
Many questions have been raised about the nature of the interaction stability between surfactants and polymers. Several factors have been proposed that may help identify the mechanisms and forces that drive the formation of these complexes [9]. The most "popular" interpretation refers to the hydrophobic character of both the surfactant and sections of the polymer chains [10].

Biosurfactants have some characteristics that are advantages compared to conventional surfactants. For example, biosurfactants have low toxicity, are produced from renewable substrates and many are stable under extreme conditions, such as $\mathrm{pH}$, temperature and ionic strength. Additionally, these surfactants are biodegradable [11] [12]. Bile salts are natural surfactants produced in the gall bladder. They form small aggregates that help to solubilize and disperse hydrolyzed fats and lipids that come from food [11] [13]. During the digestion of lipids, bile salts create mixed micelles or emulsions that are responsible for the transport of some types of partially hydrolyzed fats (for example, monoglycerides) [12] [14] [15]. Unlike typical surfactants with a polar head and an alkyl chain, bile acids consist of a rigid steroid backbone (with up to three hydroxyl groups) and an extended alkyl chain of various lengths terminating in a carboxylic acid group, which can be conjugated with glycine or taurine. This difference in structure leads to atypical aggregation behavior in bile salts [11].

Studies on the micellization of bile salts have to help reveal their physiological processes, such as their interaction with biological membranes, biliary secretion, hydrolysis of cholesterol and solubilization of cholesterol. The structure of bile salts in water has been the subject of discussions among researchers since the $60 \mathrm{~s}$ [11] [13] [14] [16] [17]. Due to their particular structure and the stiffness of the bile salt molecule, the aggregation properties differ from that of classical surfactants [11] [18]-[20]. Small et al. [17] proposed the formation of primary and secondary micelles of bile salts. They concluded that only the primary micelles, of aggregation numbers up to 10 monomers, form above the critical micellar concentration (cmc), presumably through hydrophobic interactions between the nonpolar sides of the monomers [17]. These conclusions, however, have been questioned by several authors. Oakenfull and Fisher [21], disagreeing with Small, proposed that the primary micelles are formed by hydrogen bonding. Using light scattering techniques, Kratohvil et al. [22] determined the aggregation number at the cmc for different concentrations of bile salts. The results were consistent with the formation of the dimeric species through hydrogen bonding. The authors also hypothesized that, with an increase in the bile salt concentration, the formation of larger aggregates occurs through hydrophobic interactions.

The simulation and understanding of the micellization process for mixtures of amphiphilic bile salts and polymers are relevant for understanding the stabilization and the physical-chemical properties of micellar aggregates [13]. Considerable impetus was provided to research in the field by the surface tension studies of Jones [23]. He showed that, in a mixture of the surfactant SDS and the relatively weakly surface-active polymer PEO, incorporation of the PEO led to two new transitions in the surface tension/logarithm of concentration $(\gamma / \mathrm{lnc})$ plot of the surfactant, which shows two transition points in a typical profile of surface tension. The author showed that there were three regions with distinct behavior. At the first transition point, the concentration represents the concentration where the interaction between polymers and surfactant begins (called $\boldsymbol{c a c}=$ critical aggregation concentration); the second transition point represents the concentration at which the saturation of the polymer chain by surfactants occurs (pps = point of polymer saturation) [9]. Finally, above the pps, two types of aggregates coexist: the ordinary micelles of the surfactants and the polymer-micelle complexes.

Many factors control "reactivity" in mixed surfactant-polymer systems. Specifically, properties such as the surface tension of the surfactant mixtures may affect the efficiency of their interaction with polymers [24]. For an additional example, hydrophobicity (and surface activity) of the polymer can be a dominant factor in the case of uncharged polymer/ionic surfactant pairs, while the interaction of oppositely charged species can be dominant in polyelectrolyte/ionic surfactant systems. This happens because the surfactants are active surface agents. They decrease the interfacial and surface tensions through adsorption and orientation at the interface. However, not only is there no clear understanding of the specific interactions between polymer and surfactant which control bulk and surface properties, but there is not yet a satisfactory model for describing polymer/surfactant mixing at an interface and its relation to surface properties. For example, it is difficult to determine whether surfactants and polymers interact at the air/solution interface because there is no model of the surface tension behavior of such mixtures in terms of these interactions. There are models for describing the surface tension of binary surfactant solutions in terms of an effective interaction between the two surfactant species (see, e.g., Ref. [4]), but these models are not easily extended to polymer/surfactant mixtures. In general, one would expect some sort of interaction between a surfactant monomer and a polymer segment, and the sign and magnitude of such an interaction must affect the surface properties, especially below the cac. 
This property arises because the surfactants are active surface agents, which decrease the interfacial and surface tensions due to adsorption and orientation on the interface.

Dal-Bó and collaborators [7] observed that another hydrophobically modified polymer, ethyl (hydroxyethyl) cellulose (EHEC), demonstrated a strong time dependency of adsorption at the air-water surface, which required some time to reach equilibrium between the monomeric forms on the surface and in the bulk. This fact is due to the flexibility of the polymer and the chemical modification of the cellulose that, depending on polarity, present different tendencies to adsorb on the interface. By using measures of the dynamic surface tension on the air-liquid interface, Nahringbauer [25] recently studied the time dependency of adsorption of the EHEC-SDS system and identified the beginning of cooperative association through a change in the dynamic adsorption of approximately $2.0 \mathrm{mM}$. Because of these observations, the objective of this work was to investigate the stabilization of water solutions of poly (ethylene oxide) (PEO) in the absence and presence of an anionic surfactant, sodium dodecyl sulfate (SDS), and the bile salt, sodium cholate (NaC). The primary objective was to monitor and interpret the variations in surface tension as a function of the stabilization time of solutions containing PEO and sodium cholate, that is, to monitor the time that the system required to reach a balance between micellar and aqueous phases. The experimental conditions used were the ones usually used in interfacial properties studies; they included measures of cac, pps and determination of the thermodynamic parameters of the neutral water-soluble polymer systems.

\section{Experimental Methods}

\subsection{Materials}

The surfactants sodium dodecyl sulfate (SDS) and sodium cholate (NaC), both with a 99\% purity, were obtained from Sigma, Brazil. The poly (ethylene oxide) (PEO), with an average molecular weight of $10.000 \mathrm{~g} / \mathrm{mol}$, was also obtained from Sigma, Brazil. These reagents were mixed without prior purification. The micellization parameters of the bile salt mixtures and anionic surfactant were determined in the absence and presence of different PEO concentrations and $20 \mathrm{mM}$ of the buffer 2-Amino-2-hydroxymethyl-propane-1,3-diol (tris)/HCI, pH 9.0 at $25.0^{\circ} \mathrm{C}$. The $\mathrm{pH}$ was determined using a Beckman model $\varphi 71 \mathrm{pH}$ meter (USA), which was equipped with a combined glass electrode. The electrode was calibrated against standard buffers in a thermostated, stirred vessel at the appropriate temperature $\left( \pm 0.1^{\circ} \mathrm{C}\right)$.

The aqueous PEO polymer solutions were prepared in concentrations varying from 0.1 to $100 \mathrm{mM}$ under continuous stirring for 12 hours. Stock solutions of the surfactant were made under continuous stirring and prepared at the appropriate concentrations through successive dilutions.

\subsection{Method-Surface Tension}

The variation of the surface tension was monitored in a tensiometer from Krüss GMBH, model K8 (Germany), equipped with a ring of Pt-Ir-20 and a scale divided into $0.1 \mathrm{mN} \cdot \mathrm{m}^{-1}$. The samples were held in a thermostated flask with a volume of $10.00 \mathrm{~mL}$. The presented values are an average of three measurements. The temperature was maintained at $25.0^{\circ} \mathrm{C} \pm 0.1^{\circ} \mathrm{C}$.

\section{Results and Discussion}

The dynamic surface tension of aqueous solutions containing surfactants of low molecular weight has been widely discussed by several researchers [5] [16] [26]-[29]. The dependence of the reduction of surface tension on time induced by a polymer molecule must involve a growth in the number of segments adsorbed at the interface per unit of area with time; the surface properties of a solution containing a polymer depend on the length and distribution of those segments.

Only a few studies have tried to examine the interaction between the polymer and the surfactant at the air/ liquid and liquid/liquid interfaces [26] [28] [29]. Likewise, the process of dynamic adsorption of polymers and mixtures of surfactants between or at the surface has similarly only been reported in a few studies. Surfactants and polymers that have surface activities (and mixtures of the two) influence the equilibration time of the surface tension and the equilibrium adsorption concentration from milliseconds to several hours.

In an aqueous solution, bile salts are known to associate and form aggregates, whose characteristics depend 
on the experimental conditions, such as the ionic strength of the medium and the concentration of the bile salt [24] [30]. At low concentrations, when compared with conventional ionic surfactants, the bile salts associate to form small, micellar aggregates. Thus, Zana and Guvelli [30] suggested a model of association for bile salt in which, with an increase of surfactant concentration, they form small micelles that had aggregation numbers on the order $\approx 16$ (for $\mathrm{NaC}$ ), which the authors called primary micelles. These aggregates grew with an increase in the concentration of surfactant and formed larger micellar aggregates called secondary micelles. Through light scattering experiments, Kratohvil and collaborators [22] determined the aggregation number of 10 monomers per aggregate at concentrations near the cmc. They concluded that the formation of dimers occurred through hydrogen bonding and that, with an increase in concentration, aggregates grew through hydrophobic interactions between dimers. These results suggest that a bile salt solution is composed of polydisperse aggregates with various numbers of molecules. Because of this type of association process, cmc is not clearly defined using conventional techniques, such as measures of the electrical conductivity of the solution. These conclusions were also reported by other authors using measures of fluorescence [31] and solubilization [32], which shows the need to follow the interaction between the micelles and polymeric chains over time; in other words, the determination of the time those aggregates would take to reach the surface equilibrium. Effect of PEO in the absence of surfacetants

\subsection{Effect of PEO in the Absence of Surfactants}

Figure 1 shows the graph of surface tension as a function of stabilization time for different concentrations of PEO. Note that as the concentration of PEO was increased, the time required to stabilize the air/solution interface also increased, which shows that the polymers took longer to arrange themselves at the solution/air interface for higher concentrations. Another important observation, presented in the insert of Figure 1 and in Table 1, is that with an increase in PEO concentration, the surface tension values decreased at the solution stabilization point. This reduction of surface tension means that there was an increase in the amount of polymer at the surface of the solution when the concentration was increased.

In Table 1, the times required for stabilization of the air/solution interface and the values of surface tension at the moment of stabilization are listed for different concentrations of PEO. Notably, with an increase in the concentration of PEO, the surface tension value decreased, which indicates that the PEO concentration was also increased at the solution surface; therefore, the surface tension was reduced.

When measurements were made in the presence of the tris/ $\mathrm{HCl}$ buffer, the stabilization time of the studied

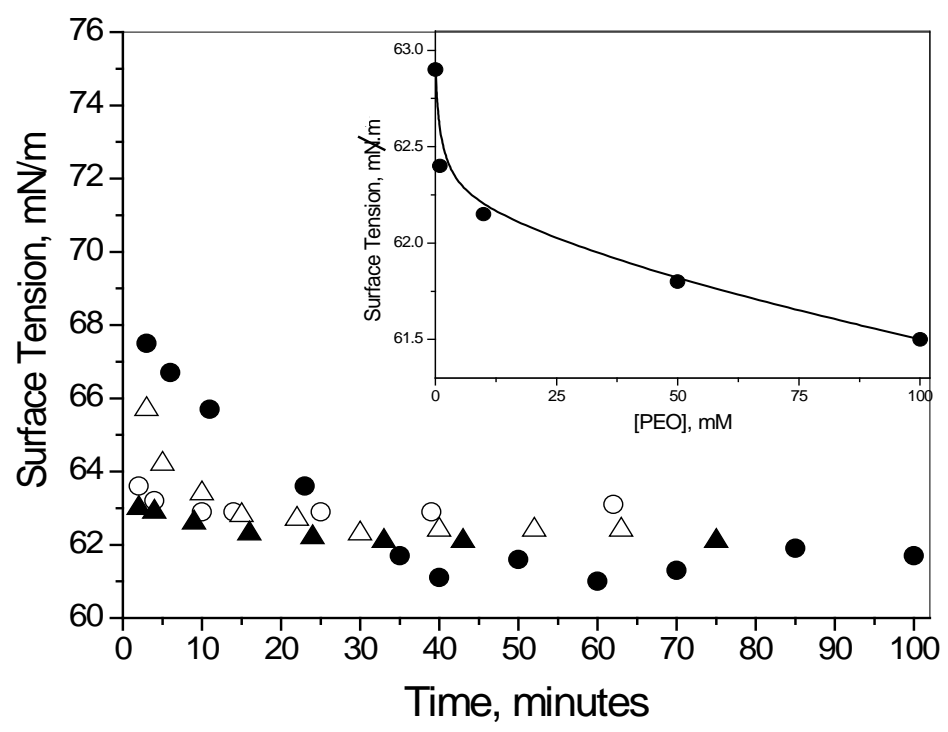

Figure 1. Profiles of the surface tension as a function of time obtained in (०) $0.1 \mathrm{mM}$; $\triangle$ ) $1.0 \mathrm{mM}$; $(\Delta) 10.0 \mathrm{mM}$ and $(\bullet) 100.0 \mathrm{mM}$ PEO. The inset shows the variation of the surface tension as a function of PEO concentration. 
Table 1. Times required for the stabilization of the air/solution interface and the values of the surface tension at the moment of stabilization for different concentrations of PEO.

\begin{tabular}{ccc}
\hline [PEO], $\mathrm{mM}$ & Surface tension $(\mathrm{mN} / \mathrm{m})$ & Stabilization time of solutions (minutes) \\
\hline 0.1 & 62.9 & 10 \\
1.0 & 62.4 & 30 \\
10.0 & 62.1 & 33 \\
50.0 & 61.8 & 35 \\
100.0 & 61.1 & 40 \\
\hline
\end{tabular}

system did not present significant variation, which we attributed to the high solubility of tris in water. The molecules of tris are strongly solvated within the solution and do not show a preference to locate at the air/solution interface and, therefore, they do not compete with the polymer. This result indicates that the use of the tris buffer is an excellent option for studies of the solution interface because it does not interfere significantly with the surface tension results. The buffer is necessary to maintain basic $\mathrm{pH}$ conditions, which results from the use of the surfactant $\mathrm{NaC}$.

\subsection{Surface Tension of the PEO and SDS Mixture in Tris/HCl Buffer}

The addition of an anionic surfactant to the PEO solution would be expected to cause a significant change in the surface tension profile as follows: (i) the surfactant associates with the polymer chains and modifies the polymer solubility at the air/solution interface and in the bulk water; (ii) the surfactant molecules compete with the polymer chains at the solution/air interface; and (iii) the effects of (i) and (ii) strongly depend on the concentrations of surfactant and polymer.

Figure 2 shows a graph of the surface tension as a function of time for a solution composed of $1.0 \mathrm{mM}$ SDS and $20 \mathrm{mM}$ PEO in tris/HCl buffer. The initial moment of stabilization was determined as the point where there was no further variation in the value of the surface tension for three subsequent readings (as seen in Figure 2). Under these conditions, approximately 60 minutes were required to stabilize the solution/air interface, which shows that the SDS monomers strongly compete with the polymer at the solution interface. Note that the concentration of surfactant used was below the cmc of SDS $8.0 \mathrm{mM}$; see the inset of Figure 3.

Table 2 shows the values of the surface tensions and respective stabilization times for different concentrations of SDS in the absence of PEO. Figure 3 shows that, with an increase in the concentration of SDS, the stabilization time at the interface of SDS solution also increases (Table 2). The surface tension varied with stabilization time until a concentration of SDS (approximately $10 \mathrm{mM}$ ); above this concentration, there was almost no further variation in the studied property (Figure 3). The inset of Figure 3 is the profile of the surface tension as a function of log SDS concentration. This plot has an inflection point at $8 \mathrm{mM}$, which indicates that any surfactant added after this concentration, known as the cmc (critical micellar concentration), heads for the solution interior; starting at this surfactant concentration, the formation of regular micelles occurred. Once the cmc was reached, the stabilization time of the surface tension was instantaneous, with the excess added surfactant forming micelles that grew with the increase of SDS concentration; therefore, they no longer competed with the polymer at the water/air interface.

\subsection{Surface Tension of the PEO and NaC Mixture in Tris/HCl Buffer}

Figure 4 shows the profile of the surface tension as a function of time for a solution composed of $2.0 \mathrm{mM} \mathrm{NaC}$ and $20.0 \mathrm{mM}$ PEO. The time required to stabilize the interface of this solution was larger than all of the ones observed in Figure 1, which only had PEO. Due to the hydrophobicity of the surfactants, both NaC and SDS compete with the polymer at the solution surface; as soon as they are added, they move to the solution/air interface and repel the water molecules.

The inset of Figure 4 is a profile of surface tension as a function of $\mathrm{NaC}$ concentration and $20 \mathrm{mM}$ PEO. The cmc of the bile salt was $10 \mathrm{mM} \mathrm{NaC}$. The stabilization time observed for concentrations greater than the cmc was immediate (the same as for the PEO/SDS system described earlier). Table 3 shows the values of the surface tension and the respective stabilization times for the different concentrations of $\mathrm{NaC}$. The results show that, for 


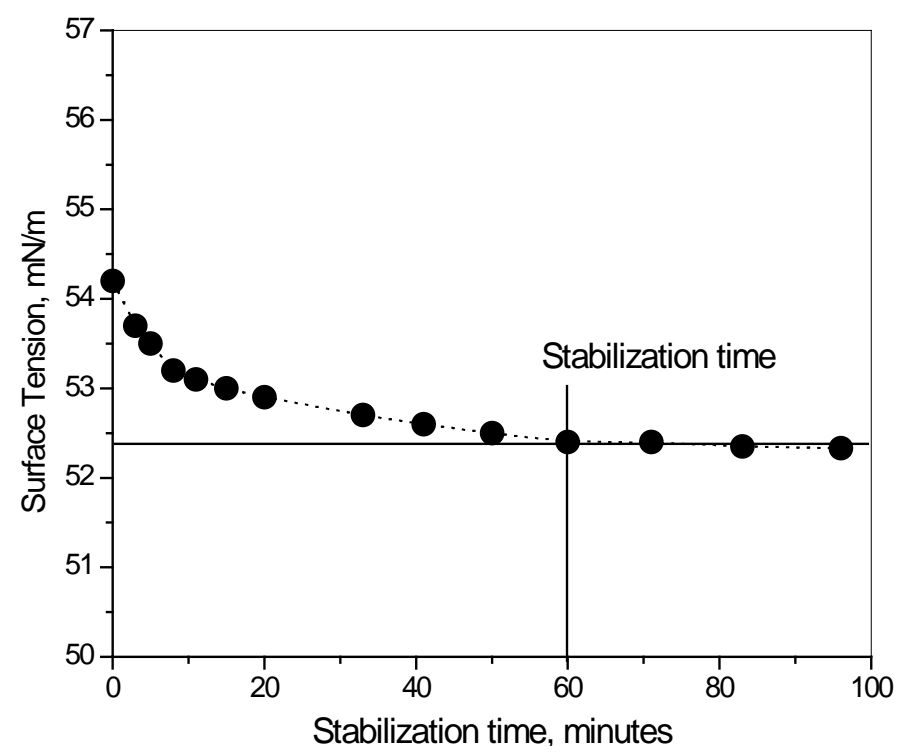

Figure 2. Graph of the surface tension as a function of time for 1.0 $\mathrm{mM}$ of SDS in the presence of $20.0 \mathrm{mM}$ PEO and TRIS/HCI buffer.

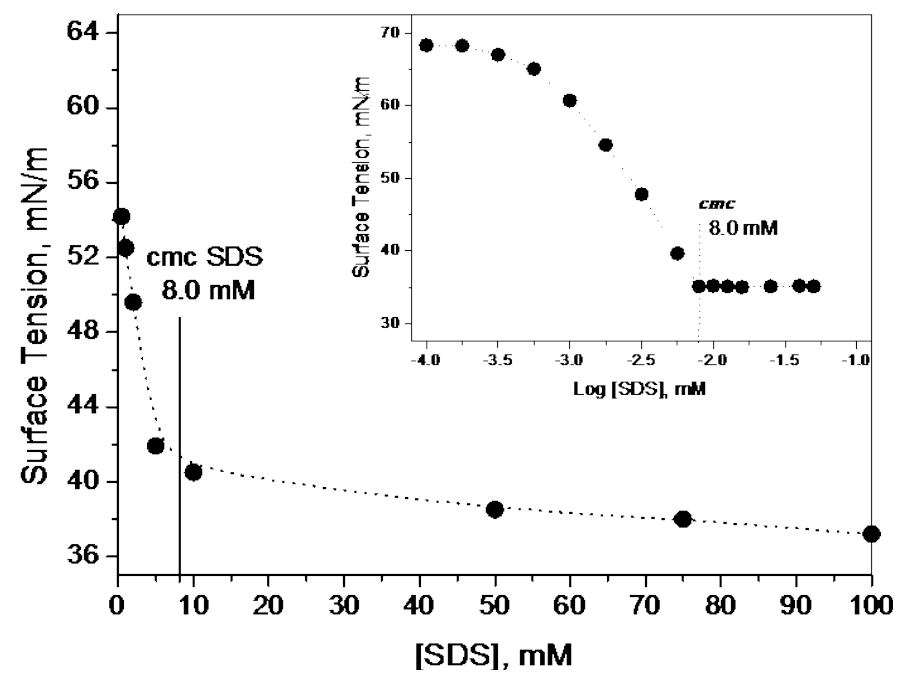

Figure 3. Variation of the value of surface tension (after stabilization) as a function of SDS concentration. The inset in Figure 3 is a profile of the surface tension as function of $\log$ [SDS].

Table 2. Values of the surface tension and the respective stabilization times for different SDS concentrations in the absence of PEO.

\begin{tabular}{ccc}
\hline [SDS], $\mathrm{mM}$ & Surface tension $(\mathrm{mN} / \mathrm{m})$ & Stabilization time of solutions (minutes) \\
\hline 0.5 & 54.2 & 13 \\
1.0 & 52.4 & 60 \\
5.0 & 41.7 & 65 \\
10.0 & 40.5 & 0 \\
50.0 & 38.5 & 0 \\
75.0 & 38.3 & 0 \\
100.0 & 37.2 & 0 \\
\hline
\end{tabular}


Table 3. Values of the surface tension and the respective stabilization times for different concentrations of NaC.

\begin{tabular}{ccc}
\hline$[\mathrm{NaCl}], \mathrm{mM}$ & Surface tension $(\mathrm{mN} / \mathrm{m})$ & Stabilization time of solutions (minutes) \\
\hline 0.5 & 60.8 & 25 \\
1.0 & 59.2 & 52 \\
5.0 & 54.6 & 72 \\
10.0 & 52.8 & 0 \\
50.0 & 47.9 & 0 \\
75.0 & 49.9 & 0 \\
\hline
\end{tabular}

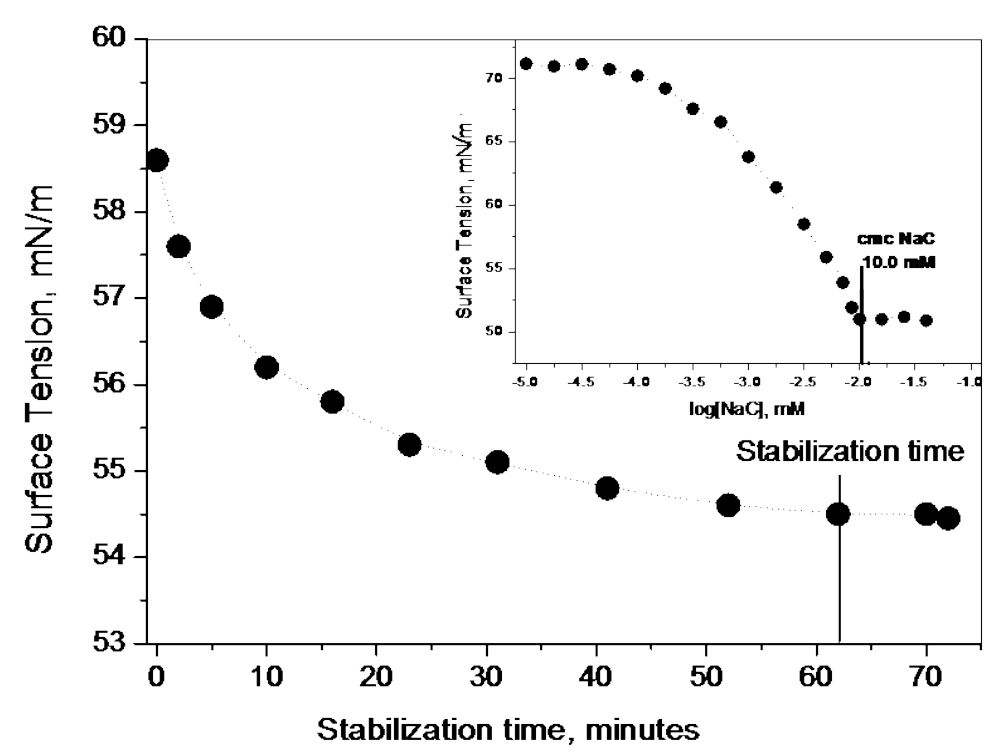

Figure 4. Surface tension as a function of time for the stabilization of a solution composed of $2.0 \mathrm{mM} \mathrm{NaC}$ and $20 \mathrm{mM}$ PEO. The insert shows the variation of surface tension as a function of $[\mathrm{NaC}]$.

concentrations lower than $5 \mathrm{mM} \mathrm{NaC}$, the surface tension had a strong time dependence on stabilization, as was also demonstrated for the surfactant SDS.

\subsection{Surface Tension of PEO with Various Mole Fractions of Mixed NaC and SDS in Buffer Tris/HCI}

All of the SDS/NaC mole fractions analyzed in the presence of $20 \mathrm{mM}$ PEO showed variation in the surface tension values (Figure 5). The total concentrations of the mole fractions of the mixed surfactants were below the cmc of each pure surfactant. For low fractions of $\mathrm{NaC}$ (up to $20 \% \mathrm{NaC}$ ), no significant variation was observed in the surface tension. However, with larger fractions of $\mathrm{NaC}$, they grew substantially. This result is consistent with the fact that $\mathrm{NaC}$ is a surfactant with surface activity inferior to SDS, and it does not locate itself as effectively on the surface; consequently, $\mathrm{NaC}$ does not cause such a marked effect at the air/solution interface. This effect is easily seen in the inset of Figure 3, where the cmc of SDS is $35 \mathrm{mN} \cdot \mathrm{m}^{-1}$, and in the insert of Figure 4, where the $\mathrm{NaC}$ had a cmc of $51 \mathrm{mN} \cdot \mathrm{m}^{-1}$.

The stabilization times presented for the different fractions of surfactant were identical; that is, a higher quantity of one or the other amphiphilic surfactant does not change the equilibrium time of the solution, only the values of surface tension. This fact may be explained by the fact that both surfactants have very similar cmc values, which makes both compete with PEO until a concentration of approximately $10 \mathrm{mM}$, at which point the micellar formation process begins. 


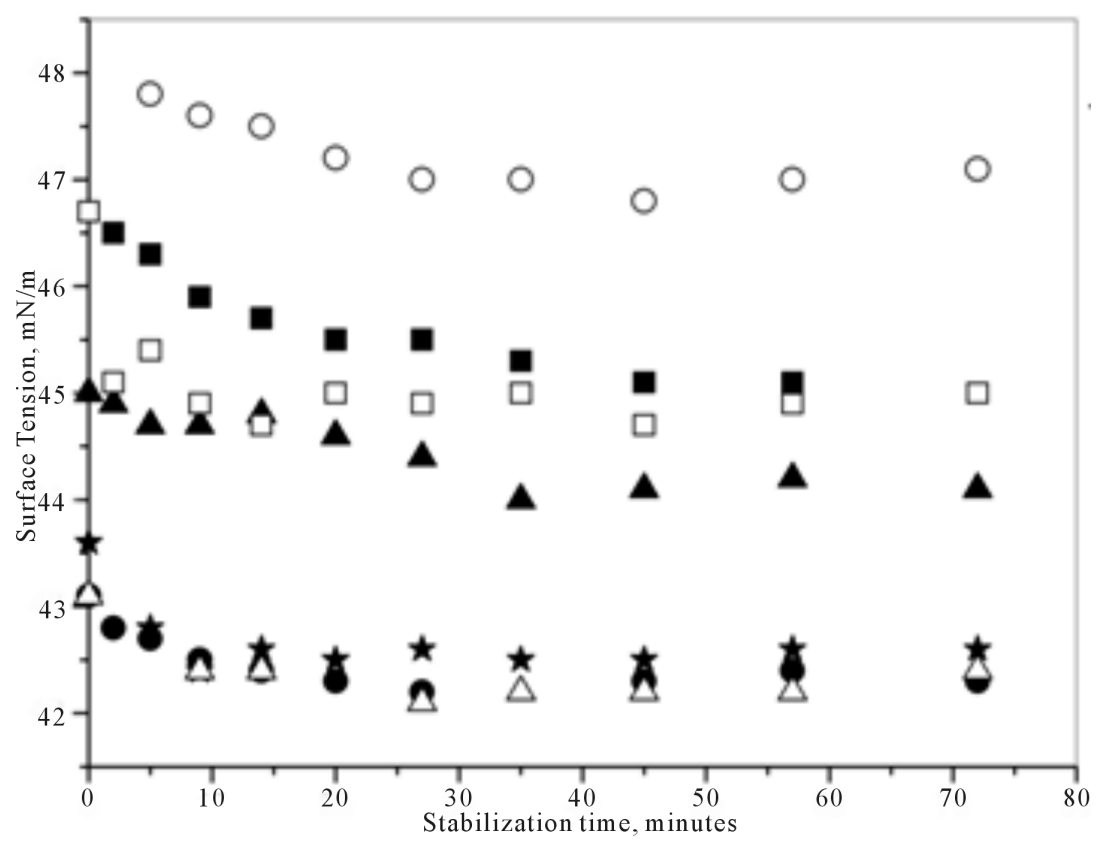

Figure 5. Profile of the surface tension as a function of the settling time by varying the $\mathrm{NaC}$ fraction in NaC/SDS $(\bullet) 5 \%$, (*) $10 \%,(\triangle) 20 \%,(\boldsymbol{\Delta}) 50 \%,(\square) 75 \%$, (口) $80 \%$ and $(O) 90 \%$ in the presence of $20 \mathrm{mM}$ of PEO.

\section{Conclusions}

Many techniques have been used to characterize the self-assembly phenomena between a polymer and surfactant in water. The product of the association process is important because these components often cause changes in the physico-chemistry stability, rheology, etc., and because many different areas of industry use them for practical purposes.

Monitoring the surface tension led to the conclusion that solutions of PEO required time to reach equilibrium and that this time depended mainly on the polymer concentration. The more concentrated the solution, the longer the time required to stabilize and the lower the surface tension value. This behavior was also observed for solutions of $\mathrm{PEO}$ in tris/HCl buffer because tris does not influence the variation in surface tension and the stabilization time of solutions.

For solutions with mixtures of PEO and SDS in buffer, it was observed that the stabilization time increased due to the surfactant molecules competing with the polymer monomers at the surface. The surface tension varied up to a concentration of $5.0 \mathrm{mM}$. Over $10.0 \mathrm{mM}$, the micellization process was rapid because those concentrations are greater than the surfactant cmc. Solutions of PEO in the presence of $\mathrm{NaC}$ show the same behavior as the PEO/SDS system; that is, at concentrations greater than the cmc, they have an immediate stabilization time.

In the case of solutions containing PEO and mixtures of SDS and $\mathrm{NaC}$, the stabilization times were very similar. An increase in surface tension was only observed for high fractions of $\mathrm{NaC}$ (greater than 0.50 ).

Finally, these results serve as a reference to know the minimum aging time of solutions needed before one should initiate any measurements of surface tension. Because the surface tensions of the PEO solutions are time dependent, all solutions in further studies are maintained at rest for at least 60 min before use.

\section{Acknowledgements}

The authors wish to thank Conselho Nacional de Pesquisa e Desenvolvimento (CNPq-Brazil) for financial support.

\section{References}

[1] Taylor, D.J.F., Thomas, R.K. and Penfold, J. (2007) Polymer/Surfactant Interactions at the Air/Water Interface. Ad- 
vances in Colloid and Interface Science, 132, 69-110. http://dx.doi.org/10.1016/j.cis.2007.01.002

[2] Claesson, P.M., Makuska, R., Varga, I., Meszaros, R., Titmuss, S., Linse, P., Pedersen, J.S. and Stubenrauch, C. (2010) Bottle-Brush Polymers: Adsorption at Surfaces and Interactions with Surfactants. Advances in Colloid and Interface Science, 155, 50-57. http://dx.doi.org/10.1016/j.cis.2010.01.004

[3] Zanette, D., Ruzza, A.A., Froehner, S.J. and Minatti, E. (1996) Polymer-Surfactant Interactions Demonstrated by a Kinetic Probe: Degree of Ionization. Colloids and Surfaces, 108, 91-100. http://dx.doi.org/10.1016/0927-7757(95)03355-6

[4] Zanette, D., Froehner, S.J., Minatti, E. and Ruzza, A.A. (1997) Effects of Polymer and Salt Concentrations on Ketal Acid Hydrolysis in Solutions of Sodium Dodecyl Sulfate and Poly(Vinyl Pyrrolidone) or Poly(Ethylene Oxide). Langmuir, 13, 659-665. http://dx.doi.org/10.1021/la960557l

[5] Dal Bo, A., Schweitzer, B., Felippe, A.C., Zanette, D. and Lindman, B. (2005) Ethyl(Hydroxyethyl)Cellulose-Sodium Dodecanoate Interaction Investigated by Surface Tension and Electrical Conductivity Techniques. Colloid Surface A, 256, 171-180.

[6] Minatti, E. and Zanette, D. (1996) Salt Effects on the Interaction of Poly(Ethylene Oxide) and Sodium Dodecyl Sulfate Measured by Conductivity. Colloid Surface A, 113, 237-246. http://dx.doi.org/10.1016/0927-7757(96)03573-X

[7] Dal-Bó, A.G., Laus, R., Felippe, A.C., Zanette, D. and Minatti, E. (2011) Association of Anionic Surfactant Mixed Micelles with Hydrophobically Modified Ethyl(Hydroxyethyl)Cellulose. Colloid Surface A, 380, 100-106. http://dx.doi.org/10.1016/j.colsurfa.2011.02.028

[8] Dal-Bó, A.G. and Minatti, R.L.e.E. (2011) Autoassociação de misturas dos Surfactantes Dodecanoato de Sódio (SDoD) e Decanoato de Sódio (SDeC) com o Polímero Hidrofobicamente Modificado Etil(Hidroxietil)Celulose (EHEC). Química Nova, 34, 1001-1006. http://dx.doi.org/10.1590/S0100-40422011000600017

[9] Goddard, E.D. and Ananthapadmanabhan, K.P. (1993) Protein-Surfactant Interactions. In: Goddard, E.D. and Ananthapadmanabhan, K.P., Eds., Interactions of Surfactants with Polymers and Proteins, CRC Press, Boca Raton, 319365.

[10] Holmberg, K., Jonsson, B., Holmberg, K. and Lindman, B. (2002) Surfactants and Polymers in Aqueous Solution. John Wiley \& Sons, New York. http://dx.doi.org/10.1002/0470856424

[11] Gouin, S. and Zhu, X.X. (1998) Fluorescence and NMR Studies of the Effect of a Bile Acid Dimer on the Micellization of Bile Salts. Langmuir, 14, 4025-4029. http://dx.doi.org/10.1021/la971155w

[12] Hildebrand, A., Neubert, R., Garidel, P. and Blume, A. (2002) Bile Salt Induced Solubilization of Synthetic Phosphatidylcholine Vesicles Studied by Isothermal Titration Calorimetry. Langmuir, 18, 2836-2847. http://dx.doi.org/10.1021/la011421c

[13] Felippe, A.C., Schweitzer, B., Dal Bo, A.G., Eising, R., Minatti, E. and Zanette, D. (2007) Self-Association of Sodium Cholate with Poly(Ethylene Oxide) Cooperatively Induced by Sodium Dodecyl Sulfate. Colloid Surface A, 294, 247-253. http://dx.doi.org/10.1016/j.colsurfa.2006.08.019

[14] Jiang, L., Wang, K., Deng, M., Wang, Y. and Huang, J. (2008) Bile Salt-Induced Vesicle-to-Micelle Transition in Catanionic Surfactant Systems: Steric and Electrostatic Interactions. Langmuir, 24, 4600-4606. http://dx.doi.org/10.1021/la7035554

[15] Eising, R., Felippe, A.C. and Domingos, J.B. (2011) Physicochemical Investigation of the Association of the Biosurfactants Sodium Cholate and Sodium Dodecanoate with Poly(ethyleneoxide). Journal of Dispersion Science and Technology, 33, 75-82. http://dx.doi.org/10.1080/01932691.2010.530094

[16] Fernandez-Leyes, M.D., Messina, P.V. and Schulz, P.C. (2011) Bile Salt Structural Effect on the Thermodynamic Properties of a Catanionic Mixed Adsorbed Monolayer. Colloid and Polymer Science, 289, 179-191. http://dx.doi.org/10.1007/s00396-010-2336-1

[17] Small, D.M., Penkett, S.A. and Chapman, D. (1969) Studies on Simple and Mixed Bile Salt Micelles by Nuclear Magnetic Resonance Spectroscopy. Biochimica et Biophysica Acta, 176, 178-189. http://dx.doi.org/10.1016/0005-2760(69)90086-1

[18] D’Alagni, M., D’Archivio, A.A., Galantini, L. and Giglio, E. (1997) Structural Study of the Micellar Aggregates of Sodium Chenodeoxycholate and Sodium Deoxycholate. Langmuir, 13, 5811-5815. http://dx.doi.org/10.1021/la970337n

[19] Djavanbakht, A., Kale, K.M. and Zana, R. (1977) Ultrasonic Absorption and Density Studies of the Aggregation in Aqueous Solutions of Bile Acid Salts. Journal of Colloid and Interface Science, 59, 139-148. http://dx.doi.org/10.1016/0021-9797(77)90348-4

[20] Dey, T. and Das, A.R. (2012) Physicochemical Study of Bile Salt-Polymer Micellar Aggregates. Zeitschrift für Physikalische Chemie, 226, 315-326. http://dx.doi.org/10.1524/zpch.2012.0172

[21] Oakenfull, D.G. and Fisher, L.R. (1977) The Role of Hydrogen Bonding in the Formation of Bile Salt Micelles. The 
Journal of Physical Chemistry, 81, 1838-1841. http://dx.doi.org/10.1021/j100445a031

[22] Kratohvil, J.P., Hsu, W.P. and Kwok, D.I. (1986) How Large Are the Micelles of Di-Alpha-Hydroxy Bile-Salts at the Critical Micellization Concentrations in Aqueous-Electrolyte Solutions-Results for Sodium Taurodeoxycholate and Sodium Deoxycholate. Langmuir, 2, 256-258. http://dx.doi.org/10.1021/la00068a026

[23] Jones, M.N. (1967) The Interaction of Sodium Dodecyl Sulfate with Polyethylene Oxide. Journal of Colloid and Interface Science, 23, 36-42. http://dx.doi.org/10.1016/0021-9797(67)90082-3

[24] Zanette, D., Felippe, A.C., Schweitzer, B., Dal Bo, A. and Lopes, A. (2006) The Absence of Cooperative Binding in Mixtures of Sodium Cholate and Poly(Ethylene Oxide) as Indicated by Surface Tension, Steady-State Fluorescence and Electrical Conductivity Measurements. Colloid Surface A, 279, 87-95. http://dx.doi.org/10.1016/j.colsurfa.2005.12.045

[25] Nahringbauer, I. (1997) Polymer-Surfactant Interaction as Revealed by the Time Dependence of Surface Tension. The EHEC/SDS/Water System. Langmuir, 13, 2242-2249. http://dx.doi.org/10.1021/la960976i

[26] Um, S.U., Poptoshev, E. and Pugh, R.J. (1997) Aqueous Solutions of Ethyl (Hydroxyethyl) Cellulose and Hydrophobic Modified Ethyl (Hydroxyethyl) Cellulose Polymer: Dynamic Surface Tension Measurements. Journal of Colloid and Interface Science, 193, 41-49. http://dx.doi.org/10.1006/jcis.1997.4990

[27] Stubenrauch, C., Albouy, P.A., von Klitzing, R. and Langevin, D. (2000) Polymer/Surfactant Complexes at the Water/Air Interface: A Surface Tension and X-Ray Reflectivity Study. Langmuir, 16, 3206-3213. http://dx.doi.org/10.1021/la991277j

[28] Djuve, J., Pugh, R.J. and Sjoblom, J. (2001) Foaming and Dynamic Surface Tension of Aqueous Polymer/Surfactants Solutions 1: Ethyl(Hydroxyethyl) Cellulose and Sodium Dodecyl Sulphate. Colloid Surface A, 186, 189-202. http://dx.doi.org/10.1016/S0927-7757(00)00787-1

[29] Nahringbauer, I. (1995) Dynamic Surface Tension of Aqueous Polymer Solutions, I: Ethyl(Hydroxyethyl)Cellulose (BERMOCOLL cst-103). Journal of Colloid and Interface Science, 176, 318-328. http://dx.doi.org/10.1006/jcis.1995.9961

[30] Zana, R. and Guveli, D. (1985) Fluorescence Probing Study of the Association of Bile Salt in Aqueous Solutions. The Journal of Physical Chemistry, 89, 1687-1690. http://dx.doi.org/10.1021/j100255a028

[31] Minatti, E., Norwood, D.P. and Reed, W.F. (1998) Surfactant/Polymer Assemblies. 2. Polyelectrolyte Properties. Macromolecules, 31, 2966-2971. http://dx.doi.org/10.1021/ma971319f

[32] Sugioka, H. and Moroi, Y. (1998) Micelle Formation of Sodium Cholate and Solubilization into the Micelle. Biochimica et Biophysica Acta (BBA)_Lipids and Lipid Metabolism, 1394, 99-110. http://dx.doi.org/10.1016/S0005-2760(98)00090-3 